\title{
Compensation of MEMS Gyroscope Random Error Based on Square- Root Risk-Sensitive Unscented Kalman Filter
}

\author{
Shi Gang ${ }^{1,2,3}$, Li Xisheng ${ }^{1,4+}$, Li Ruibin ${ }^{5}$, Wang Zhe ${ }^{1}$, Kang Ruiqing ${ }^{1}$ and Shu Xiongying ${ }^{1}$ \\ ${ }^{1}$ School of Automation and Electrical Engineering, University of Science and Technology Beijing, Beijing \\ 100083, China. \\ ${ }^{2}$ College of Information and Control Engineering, China University of Petroleum, Qingdao, 266580, China. \\ ${ }^{3}$ Shengli college China University of Petroleum, Dongying 257061, China. \\ ${ }^{4}$ Beijing Engineering Research Center of Industrial Spectrum Imaging, Beijing 100083, China. \\ ${ }^{5}$ School of Electronic and Information Engineering, Beijing University of Aeronautics and Astronautics, \\ Beijing 100191, China.
}

\begin{abstract}
In order to compensate MEMS gyroscope random error, a new method employing the squareroot risk-sensitive unscented Kalman filter (SR-RSUKF) and a nonlinear model is proposed. The nonlinear model based on ARIMA takes model parameters as states, and thus realizes the online model estimation. The SR-RSUKF deals with non-additive noise items through augmented state vector, and employs a square root algorithm to get well numerical stability, and improves the flexibility by extending scalar risk parameters to a risk sensitive matrix. In experiments, the raw sample data is processed with three methods using different models and filters. And the results show that the SR-RSUKF together with the nonlinear model provide a competent solution to compensate MEMS gyroscope random error.
\end{abstract}

Keywords: MEMS gyroscope, random error, Unscented Kalman Filter

\section{Introduction}

Because of small size, inexpensiveness and power efficiency, MEMS gyroscopes have been widely used in navigation and other fields [1]. But their low accuracy limits application effect greatly. MEMS gyroscope error can be divided into deterministic error and random error [2]. A method to compensate the random error is given in [3], which use linear models and the Kalman filter. In [4], a nonlinear error model is proposed and the particle filter is applied to eliminate the random error. Risk-sensitive filter [5][6] which optimize an exponential cost function has show robustness to parametric uncertainties. In this paper, we apply the nonlinear model [4] with square-root risk-sensitive unscented Kalman filter (SR-RSUKF) to compensate random error. The filter which is based on the risk-sensitive unscented Kalman filter (RSUKF) [7] has the following characters: being applicable to models with non-additive noise items, employing non-scalar risk sensitive factor [8] and applying square root algorithm which is used in the square-root unscented Kalman filter (SR-UKF) [9].

\section{The Nonlinear Model}

MEMS gyroscope random error, denoted as $x_{k}$, can be described using ARIMA model. In this paper, the degree of autoregressive, moving average and difference operation are all chosen as one. Then, $x_{k}$ is written as:

\footnotetext{
+ Corresponding author. Tel.: + 86-10-62332018.

E-mail address: 1xs@ustb.edu.cn
} 


$$
x_{k}=(1+\varphi) x_{k-1}-\varphi x_{k-2}+a_{k}-\theta a_{k-1}
$$

where $\varphi$ is the autoregressive coefficient, $\theta$ is the moving average coefficient, and $a_{k}$ is the white noise. Let $a_{k}$ be normally distributed: $a_{k} \sim N(0,1)$, then equation (1) can be represented as:

$$
x_{k}=(1+\varphi) x_{k-1}-\varphi x_{k-2}+\delta^{1} a_{k}-\delta^{2} a_{k-1}
$$

where $\delta^{1}$ and $\delta^{2}$ are coefficients whose value depend on the error character. Hence, the state and measurement equations can be written as:

$$
\begin{aligned}
& X_{k}=\left[\begin{array}{cc}
1+\varphi & -\varphi \\
1 & 0
\end{array}\right] X_{k-1}+\left[\begin{array}{cc}
\delta^{1} & -\delta^{2} \\
0 & 0
\end{array}\right] W_{k-1} \\
& Z_{k}=\left[\begin{array}{ll}
1 & 0
\end{array}\right] X_{k}+V_{k}
\end{aligned}
$$

where $X_{k}=\left[\begin{array}{ll}x_{k} & x_{k-1}\end{array}\right]^{T}, W_{k-1}=\left[\begin{array}{ll}a_{k} & a_{k-1}\end{array}\right]^{T}$, and the process noise $W$ and measurement noise $V$ are assumed to be uncorrelated and normally distributed with covariance $Q$ and $R$ respectively.

Obviously, equation (3) and (4) compose a linear model for random error, which is named as model L in this paper. The Kalman filter can be applied using model $\mathrm{L}$ to process random error. In fact, the model parameters are affected by the environment where the gyroscope is working. To improve adaptive performance of the random error model, the model parameters are regarded as state variables [4], resulting in a new state vector $X_{k}=\left[\begin{array}{llllll}x_{k} & x_{k-1} & \varphi_{k} & \delta_{k}^{1} & \delta_{k}^{2}\end{array}\right]^{T}$. The corresponding new model is represented as:

$$
\begin{aligned}
& X_{k}=\left[\begin{array}{c}
\left(1+\varphi_{k-1}\right) x_{k-1}-\varphi_{k-1} x_{k-2} \\
+\delta_{k-1}^{1} a_{k}-\delta_{k-1}^{2} a_{k-1} \\
x_{k-1} \\
\varphi_{k-1} \\
\delta_{k-1}^{1} \\
\delta_{k-1}^{2}
\end{array}\right] \\
& Z_{k}=\left[\begin{array}{lllll}
1 & 0 & 0 & 0 & 0
\end{array}\right] X_{k}+V_{k}
\end{aligned}
$$

Compared with model L, the new model, named as model NL, realizes online estimation of model parameters. However, it's a nonlinear model with non-additive noise items.

\section{Square-Root Risk-Sensitive Unscented Kalman Filter}

Risk-sensitive filter has show more robustness to parametric uncertainties than risk-neutral filter. The fundamental difference between risk-sensitive filter and Kalman filter, which contribute to the robustness, is that the former minimizes an exponential cost criterion rather than the variance of estimation error. The exponential cost criterion is:

$$
C\left(\hat{\Phi}_{1}, \ldots \hat{\Phi}_{k}\right)=E\left(\exp \left(\mu_{1} \sum_{i=1}^{k-1} \rho_{1}\left(\Phi\left(X_{i}\right)-\hat{\Phi}_{i}^{*}\right)+\mu_{2} \rho_{2}\left(\Phi\left(X_{k}\right)-\hat{\Phi}_{k}^{*}\right)\right)\right)
$$

where $\Phi$ is the function to be estimated and its argument is the state variables $\mathrm{X}$, the subscript $\mathrm{k}$ denote the instant, $\hat{\Phi}$ and $\hat{\Phi}^{*}$ denote the estimate and optimal value respectively, $\mu_{1}$ and $\mu_{2}$ are risk parameters, and functions $\rho_{1}$ and $\rho_{2}$ are both strictly convex, continuous and bounded from below, attaining global minima at 0 . The minimum risk-sensitive estimate is defined by

$$
\hat{\Phi}_{k}^{*}=\arg \min C\left(\hat{\Phi}_{1}^{*}, \ldots, \hat{\Phi}_{k-1}^{*}, \hat{\Phi}_{k}\right)
$$

If the state variables themselves are to be estimated and both $\rho_{1}$ and $\rho_{2}$ are quadratic functions of vectors in the form $\rho(\alpha)=\alpha^{T} \alpha$, the recursion form of risk-sensitive filter for prior estimation [7] can be written as:

$$
\begin{gathered}
\sigma_{k+1 \mid k}\left(X_{k+1}\right)=\int f\left(X_{k+1} \mid X_{k}\right) g\left(Z_{k} \mid X_{k}\right) \times \exp \left(\mu_{1}\left(X_{k}-\hat{X}_{k \mid k-1}\right)^{T}\left(X_{k}-\hat{X}_{k \mid k-1}\right)\right) \times \sigma_{k \mid k-1}\left(X_{k}\right) d X_{k} \\
\hat{X}_{k \mid k-1}=\arg \min \int \exp \left(\mu_{2}\left(X_{k}-\alpha\right)^{T}\left(X_{k}-\alpha\right)\right) \times \sigma_{k \mid k-1}\left(X_{k}\right) d X_{k}
\end{gathered}
$$

where $\hat{X}_{k \mid k-1}$ is the optimal estimate, $\sigma_{k+1 \mid k}$ represents an information state [5], $\alpha$ is a parameter, and $f\left(X_{k+1} \mid X_{k}\right)$ and $g\left(Z_{k} \mid X_{k}\right)$ are probability density functions: $f\left(X_{k+1} \mid X_{k}\right)=P_{k+1 \mid k}\left(. \mid X_{k}\right)$, $g\left(Z_{k} \mid X_{k}\right)=P_{k \mid k}\left(. \mid X_{k}\right)$. 
As the integral in (9) is intractable, the RSUKF, an approximate version of the recursion, is given in [7], which is based on the UT Transform and some assumptions. In order to expand applicable scope of the RSUKF and enhance its performance, the following improvements are made: firstly, an augmented state vector is employed to deal with the non-additive noise items; secondly, to get better numerical stability, the SR-UKF is used for reference, which utilize QR decomposition and Cholesky factor updating to guaranteed positive semi-definiteness of the state covariance; lastly, the scalar risk parameter is replaced by a risk sensitive matrix in diagonal form, which can improve the flexibility of application. The new algorithm, named as SR-RSUKF, is described as follow.

(1) Filter initialization:

$$
\left\{\hat{X}_{0}=\left[\hat{X}_{0}^{r}, 0\right]^{T}, P_{0}=\operatorname{diag}\left(P_{0}^{r}, Q\right), S_{0}=\operatorname{chol}\left(P_{0}\right)\right\}
$$

where $\hat{X}_{0}, \hat{X}_{0}^{r}$ and 0 are initial values of the augmented state vector, real state vector and noise items respectively, $P_{0}, P_{0}^{r}$ and $Q$ are the corresponding initial covariances, $\operatorname{chol}()$ realize the Cholesky decomposition and return the lower triangular matrix.

For $k \in\{1, \ldots, \infty\}$ :

(2) Time updating:

Calculate sigma points $\chi_{k-1}$ with $\hat{X}_{k-1}$ and $S_{k-1}$ :

$$
\chi_{k-1}=\left[\begin{array}{lll}
\hat{X}_{k-1} & \hat{X}_{k-1}+\gamma S_{k-1} & \hat{X}_{k-1}-\gamma S_{k-1}
\end{array}\right]
$$

Update them through process dynamics $\mathrm{F}$ :

$$
\chi_{i, k \mid k-1}=F\left(\chi_{i, k-1}\right), i=0, \ldots, 2 n
$$

Get the prior estimation:

$$
\left\{\begin{array}{l}
\hat{X}_{k \mid k-1}=\sum_{i=0}^{2 n} W_{i}^{m} \chi_{i, k \mid k-1} \\
S_{k \mid k-1}=q r\left(\sqrt{W_{1}^{c}}\left[\chi_{1: 2 n, k \mid k-1}-\hat{X}_{k \mid k-1}\right]\right), S_{k \mid k-1}=\operatorname{cholupdate}\left(S_{k \mid k-1}, \chi_{0, k \mid k-1}, W_{0}^{c}\right)
\end{array}\right.
$$

where $\lambda=\alpha^{2}(n+\kappa)-n, \gamma=\sqrt{n+\lambda}, W_{0}^{m}=\lambda / \gamma^{2}, W_{0}^{c}=W_{0}^{m}+\left(1-\alpha^{2}+\beta\right), W_{i}^{m}=W_{i}^{c}=1 /\left(2 \gamma^{2}\right)(i=1, \ldots, 2 n), \alpha, \beta$ and $\kappa$ are UT Transform parameters, $\mathrm{n}$ is the dimension of $\hat{X}_{k-1}, \operatorname{qr}()$ realize QR decomposition and return the transpose of the upper triangular part of the right factor, and cholupdate() realize the rank 1 update of Cholesky factor.

(3) Risk-sensitive updating:

$$
\left\{\begin{array}{l}
S_{k \mid k-1}=\operatorname{chol}\left(S_{k \mid k-1}^{T}{ }^{-1} S_{k \mid k-1}^{-1}\right) \\
S_{k \mid k-1}=\operatorname{cholupdate}\left(S_{k \mid k-1}, \operatorname{chol}\{2 \mu\},-1\right) \\
S_{k \mid k-1}=\operatorname{chol}\left(S_{k \mid k-1}^{T}{ }^{-1} S_{k \mid k-1}^{-1}\right)
\end{array}\right.
$$

where $\mu=\operatorname{diag}\left(\mu_{1}, \ldots, \mu_{n}\right)$ is the risk sensitive matrix.

(4) Measurement updating

Recalculate sigma points $\chi_{k \mid k-1}$ with $\hat{X}_{k \mid k-1}$ and $S_{k \mid k-1}$ :

$$
\chi_{k \mid k-1}=\left[\begin{array}{lll}
\hat{X}_{k \mid k-1} & \hat{X}_{k \mid k-1}+\gamma S_{k \mid k-1} & \hat{X}_{k-1}-\gamma S_{k \mid k-1}
\end{array}\right]
$$

Update them through measurement equation $\mathrm{H}$ :

$$
Z_{i, k \mid k-1}=H\left(\chi_{i, k \mid k-1}\right), \quad i=0, \ldots, 2 n
$$

Calculate the mean and covariance:

$$
\left\{\begin{array}{l}
\hat{Z}_{k \mid k-1}=\sum_{i=0}^{2 n} W_{i}^{m} Z_{i, k \mid k-1} \\
S_{Z}=\operatorname{qr}\left(\left[\sqrt{W_{1}^{c}}\left[Z_{1: 2 n, k \mid k-1}-\hat{Z}_{k \mid k-1}\right] \sqrt{R}\right]\right), S_{Z}=\operatorname{cholupdate}\left(S_{Z}, Z_{0, k \mid k-1}, W_{0}^{c}\right) \\
P_{X Z}=\sum_{i=0}^{2 n} W_{i}^{c}\left[\chi_{i, k \mid k-1}-\hat{X}_{k \mid k-1}\right]\left[Z_{i, k \mid k-1}-\hat{Z}_{k \mid k-1}\right]^{T}
\end{array}\right.
$$

Calculate the Kalman gain: 


$$
K=P_{X Z} / S_{Z}^{T} / S_{Z}
$$

Get the posterior estimation:

$$
\left\{\hat{X}_{k}=\hat{X}_{k \mid k-1}+K\left(Z_{k}-\hat{Z}_{k \mid k-1}\right), U=P_{X Z} S_{Z}, S_{k}=\operatorname{cholupdate}\left(S_{k \mid k-1}, U,-1\right)\right\}
$$

(5) Noise states resetting:

$$
\left\{\hat{X}_{k}=\left[\hat{X}_{1: n r, k} 0\right], S_{k}=\operatorname{chol}\left(\operatorname{diag}\left(P_{1: n r, 1: n r, k}, Q\right)\right)\right\}
$$

where $n r$ is the dimension of real state vector, and $P_{k}=S_{k} S_{k}^{T}$.

\section{Experiments and Analysis}

The performances of model NL and SR-RSUKF are examined with experiments, in which raw gyroscope data is processed with three methods.

The raw data samples are collected in static condition using miniature inertial measurement unit MTi28A53G35 equipped with MEMS gyroscopes. The sampling frequency is $100 \mathrm{~Hz}$, and the duration is 60 seconds while the environment temperature range from $37.8^{\circ} \mathrm{C}$ to $40.1^{\circ} \mathrm{C}$. The methods used to compensate random error are: method 1 which employ model L and strong tracking Kalman filter (STF) [10]; method 2 which employ model NL and SR-UKF; method 3 which employ model NL and SR-RSUKF. The raw data and compensated data are plotted in figure 1. Further, the Allan variance is applied to analysis the experiment data and the results are presented in figure 2 .

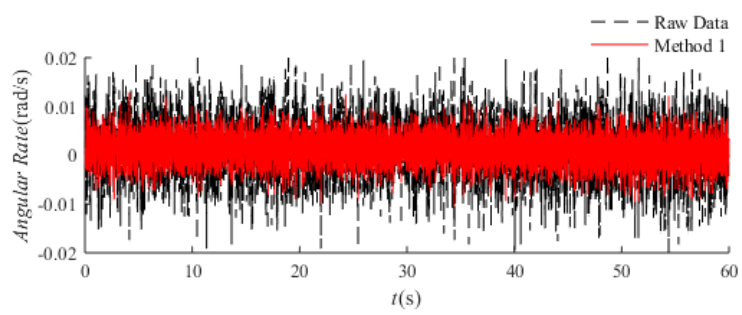

(a)

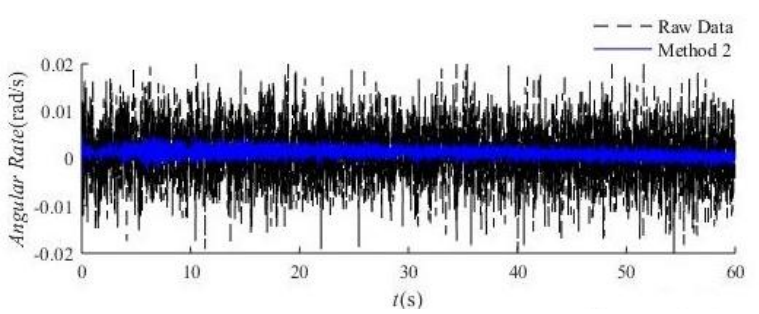

(b)

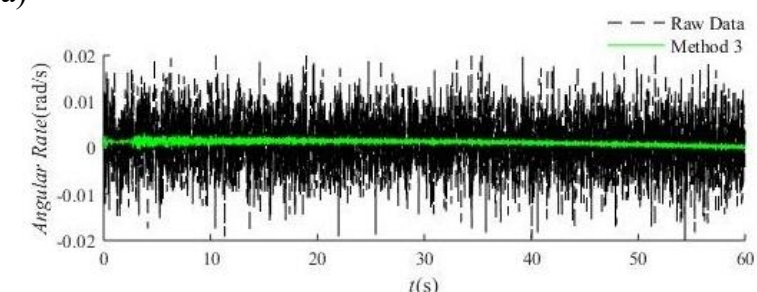

(c)

Fig. 1: (a) Raw data and compensated data with method 1. (b) Raw data and compensated data with method 2. (c) Raw data and compensated data with method 3 .

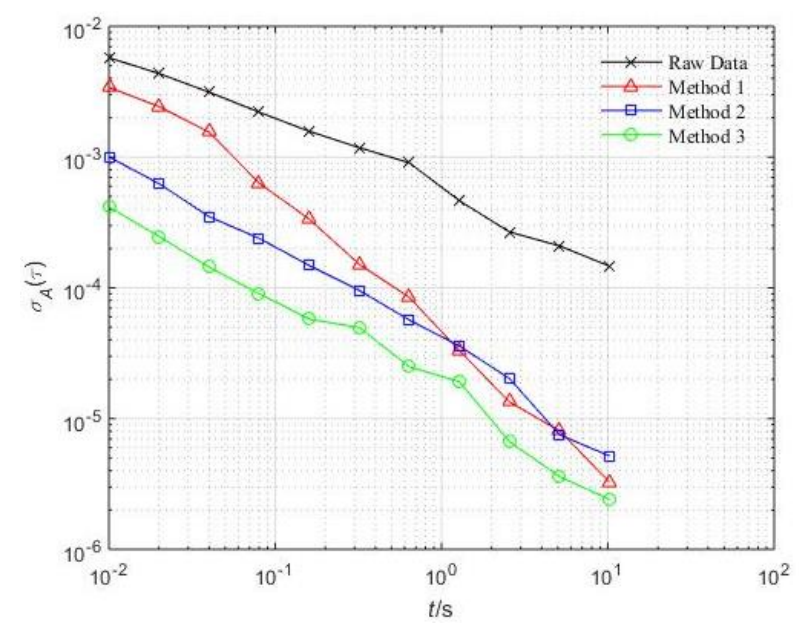

Fig. 2: Allan variance of raw data and compensated data. 
We can see from Figs. 1 and 2 that method 2 and 3 are apparently superior to method 1 . The contrast is a reflection of the difference between model L and model NL. In fact, STF has well adaptability, but the contrast suggest that the model is a more important factor, and model NL is more qualified to describe the MEMS gyroscope random error. In addition, method 3 exhibit better performance than method 2, which verify that the introduction of risk-sensitive has improved the robustness of filter.

\section{Conclusions}

Compared with the linear model, the nonlinear model which realizes online parameter estimation is more qualified to describe MEMS gyroscope random error. The SR-RSUKF based on RSUKF, can handle the nonlinear models with non-additive noise items; has well numerical stability inherited from the SR-UKF and is more robust than the later. The SR-RSUKF together with the nonlinear model provides a competent solution to compensate MEMS gyroscope random error.

\section{Acknowledgements}

This research is supported by National Natural Science Foundation of China (Project 61273082).

\section{References}

[1] Priydarshi, Raman Jaiswal, Renju C Nair, Naveen Krishna Yarlagadda, A Ashok Kumar Senapati, Prabhushetty Mulage, "Adaptive gyroscope drift compensation based on temporal noise modeling”, 2016 International Conference on Microelectronics, Computing and Communications (Micro Com), 2016, pp1-5.

[2] Zhang Yulian, Chu HaiRong, Zhang Hongwei, Zhang Mingyue, CHEN Yang, LI Yin-hai, "Characterists and compensation method of MEMS gyroscope random error", Chinese Optics, Vol.9, No.4, Aug.2016, pp501-508.

[3] Yuan Gannan, Liang Haibo, He Kunpeng, Xie Yanjun, "Analysis and Application of State Space Model for MEMS Gyro Random Drift”, CHINESE JOURNAL OF SENSORS AND ACTUATORS, Vol.24, No.6, June. 2011, pp853-858.

[4] Xiong Jian, "Research on Particle Filtering Technique and Its Application in Strap down Inertial Navigation System with Fiber Optic Gyro", Nanjing University of Aeronautics and Astronautics, College of Automation Engineering, December, 2010, pp42-47.

[5] BOEL R.K., JAMES M.R., PETERSON I.R., "Robustness and risk sensitive filtering", IEEE Trans. Automatic Control, Vol.47, No.3, Mar. 2002, pp. 451-461.

[6] BANAVAR R.N., SPEYER J.L, "Properties of risk-sensitive filters/estimators", IEE Proc. Control Theory Appl., Vol.145, No.1, Jan. 1998, pp. 106-112.

[7] S. Bhaumik ; S. Sadhu ; T.K. Ghoshal, "Risk-sensitive formulation of unscented Kalman filter", IET Control Theory \& Applications, Vol.3, No.4, April 2009, pp375-382.

[8] Arunasish Acharya, Smita Sadhu, T. K. Ghoshal, "Risk sensitive Kalman filter with non-scalar risk-sensitive factor", 2010 Annual IEEE India Conference (INDICON), 2010, pp1-4.

[9] R. Van der Merwe, E. A. Wan, "The square-root unscented Kalman filter for state andparameter-estimation" 2001 IEEE International Conference on Acoustics, Speech, and Signal Processing, 2001, pp 3461-3464.

[10] Mengyin Fu, Zhihong Deng, Jiwei Zhang, "Kalman filtering theory and its application in navigation system", Science Press, Beijing, China, Apr. 2010, pp135-139. 